\title{
Cloud-based integrated socio-sanitary care e-services in Croatia: Lessons learned
}

\author{
Igor Ljubi ${ }^{1}$, Hrvoje Belani ${ }^{2}$, and Suzana Belosevic Romac ${ }^{3}$ \\ ${ }^{1}$ VERN' University of Applied Sciences, Zagreb, Croatia \\ ${ }^{2}$ Ministry of Health, Zagreb, Croatia \\ ${ }^{3}$ City of Rijeka, Rijeka, Croatia
}

\begin{abstract}
Among many other services, modern governments offer both healthcare and social care to their citizens. Traditionally built in a silo structure and often using separate funding schemes, these two services offer limited possibilities for integration. Therefore, a novel approach is needed which will enable the integrated provisioning of both social and healthcare services to the citizens. This paper describes a platform for collaboration on multiple levels, provided to connect actors from healthcare and social care services - from primary care medical doctors to hospitals, and from home caregivers and/or family members to emergency centres for the elderly. Utilizing the cloud-based ICT tool, we have developed an integrated e-care model and introduced personalised care pathways for the elderly people suffering from various health problems (including heart failure, cardiovascular diseases, diabetes and mental health issues). This model employs a patient-centric approach to care delivery, giving the patient (or the person acting on behalf of the patient) in power to control (and to share) any data relevant to treat patient's health condition. For the socio-sanitary care providers the benefit is the ability to influence clinical outcomes by remotely monitor and coordinate care initiatives. Using this approach, government has an effective aid to meet quality thresholds, generate clinical outcome metrics and improve satisfaction of the citizens using health and social care services. Key words: integrated e-care, cloud-based services, care pathways, health issues, ICT.
\end{abstract}

\section{Introduction}

European democracies have been proud of their health care services for a long time, and it is unfortunate that those have not evolved to respond to the modern environment and are no longer fit for purpose [1]. Although they are founded on the common principles such as accessibility to quality care, equity and solidarity, in order to cope with the modern challenges, it is imperative that governments, while providing health care services, acknowledge the need for cost-efficient investments. Traditionally, social care is often underbudgeted when comparing to the health care [2], leaving the latter to bear the burden of cost savings efforts.

\subsection{Integrated socio-sanitary care}

To achieve the goals of providing good quality for both health and social services to their citizens (which is the declared programmatic concept of every modern European government) [3], a novel approach was required as the general population of Europe is getting older, thus creating more need for those services, which result in excess of cost. 


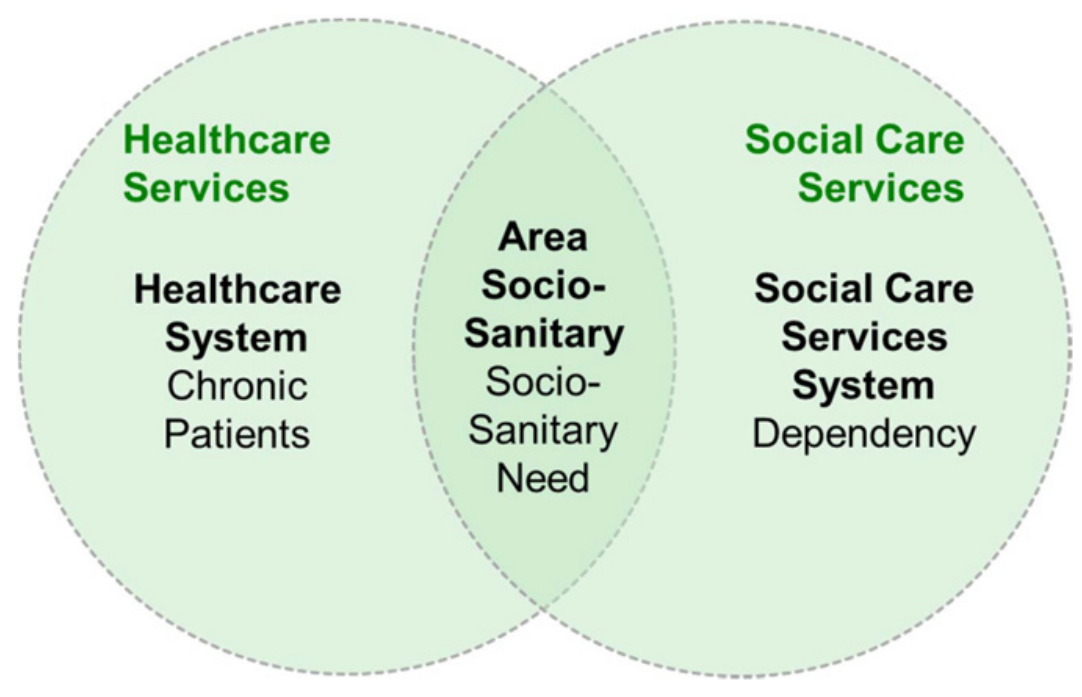

Fig. 1. Integrated socio-sanitary care.

This yielded the overarching concept of integrated socio-sanitary care [4], uniting efforts made by policy makers, medical personal, and social caregivers. Those stakeholders agree that balanced cost in delivering good quality service can be based on resource optimization, prevention (proactivity vs. reactivity) and an increased shift to out-patient treatment (care at home) is necessary [5].

Several different phrases are often used to describe similar approach to the described problem. Those include continuum of care, coordination of care, discharge planning, case management, and seamless care. It is most frequently equated with managed care in the USA, shared care in the UK, trans-mural care in the Netherlands, and other widely recognised formulations such as comprehensive care and disease management.

Despite, integrated socio-sanitary care is seen as the way forward that will benefit all Europeans (in particular older people), whilst helping to address resource efficiency and sustainability of care systems [6]. We define it as "collaboration, alignment, training and connectivity among social care, health care, and community care providers with the mission to provide better services at reduced cost".

The novelty in this concept is that it also incorporates the role of informal caregiver into the integration circle. The objective is to achieve integration between hospitals, community homes and self-care. Therewith, integrated socio-sanitary care is located at the interception of two traditional markets, the health service market and the social service market as indicated in Fig. 1.

The research presented in this paper evaluates the concept of using IT in order to facilitate various needs of an elderly person, who is suffering from chronic condition and receiving both formal and informal care. A model of integrated care pathways has been developed and was transferred into an IT tool. Several case studies aimed at validating both the approach and the IT tool itself.

Modern care requires a formation of a multi-disciplinary network, consisting of care professionals in different fields of expertise as well as informal caregivers: This network tailors their service provisioning in form of patient-centric models to the patients' care requirements. An example of such patient-centric multi-disciplinary care network is shown in Fig. 2. 


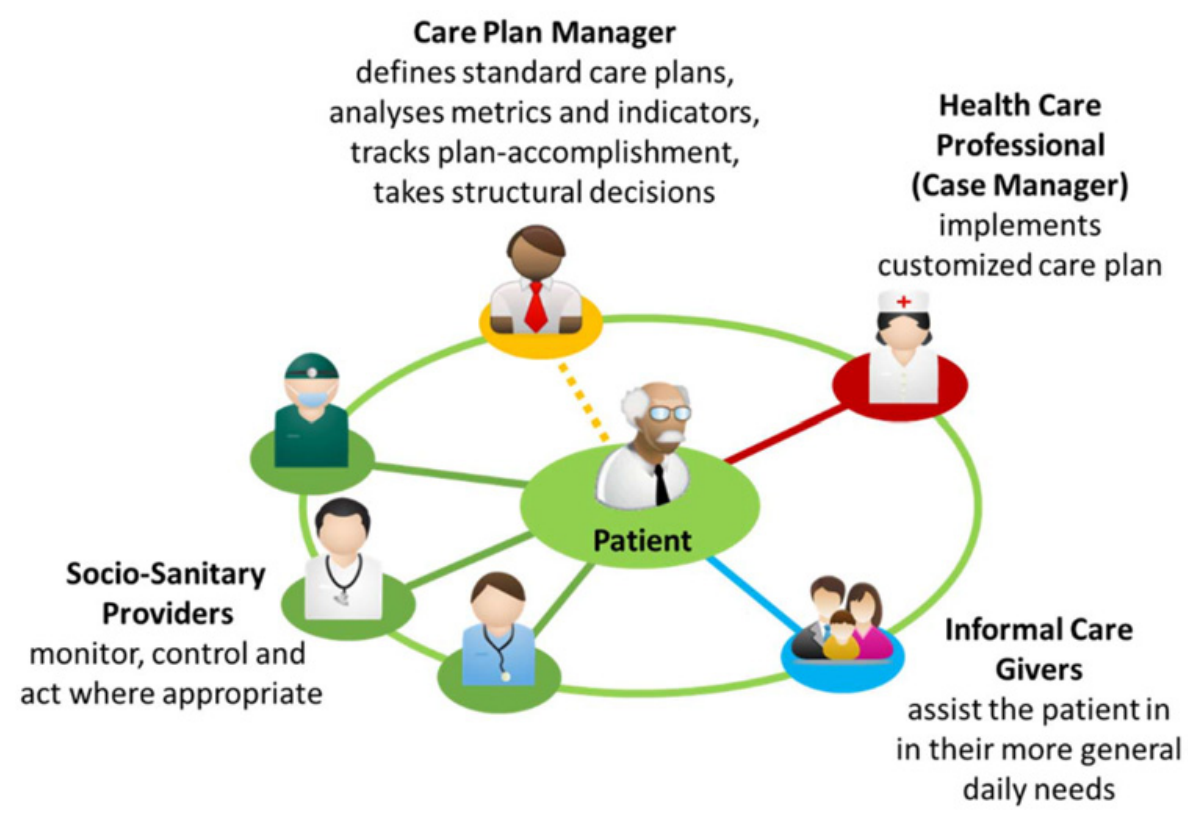

Fig. 2. Patient-centric, multi-disciplinary integrated care network.

Figure 2 depicts the patient-centric "Circle of Care" introduced and developed by the EU funded INCA project [7] that was successfully run with pilots in Spain, Cyprus, Latvia and Croatia. To enable a practical top-level management, patients are segmented and associated with standardised care plans, also called care pathways, which are then adapted by the case manager to customized integrated care plans (ICPs) to cater for each patient's individual needs.

\subsection{Social and demographic context of INCA}

Project INCA was focused on developing tools used by care providers and informal caregivers in their efforts to provide better care for the elderly citizens (over 65 and suffering from a chronic disease). INCA can be used to handle any socio-sanitary e-care service delivered also to "healthy" users, while they are not yet chronic and big "health services consumers", as a conservative way to determine who its initial target population of users will be.

However, the project sets the focus exclusively on the European elder chronic population in line with the eHealth Action Plan 2012-2020 which says "two out of three people (66.66\%), who have reached retirement age have at least two chronic conditions" [8].

According to Eurostat in the year 2012, the EU27 population was 503.663.601. The population over 65 years old was categorized as follows: ages 65-79 years represented a percentage of $12.90 \%$; while ages over 80 stood for a percentage of $4.90 \%$. Consequently, the people in Europe that are aged over 65 years represented a total percentage of $17.8 \%$ or a number of 89.652.121. Estimated projections for elder population in the EU between the years 2017-2021 show a 2.28\% growth for each year. Moreover, population over 80 years in the EU27 is projected to grow from 5\% in 2010 to $11.5 \%$ in 2050. Statistics reveal that the highest speed of ageing (in terms of median age) is most possible to happen in most of the Eastern Europe countries. 
Projected Percentage of Population Aged 65 and over

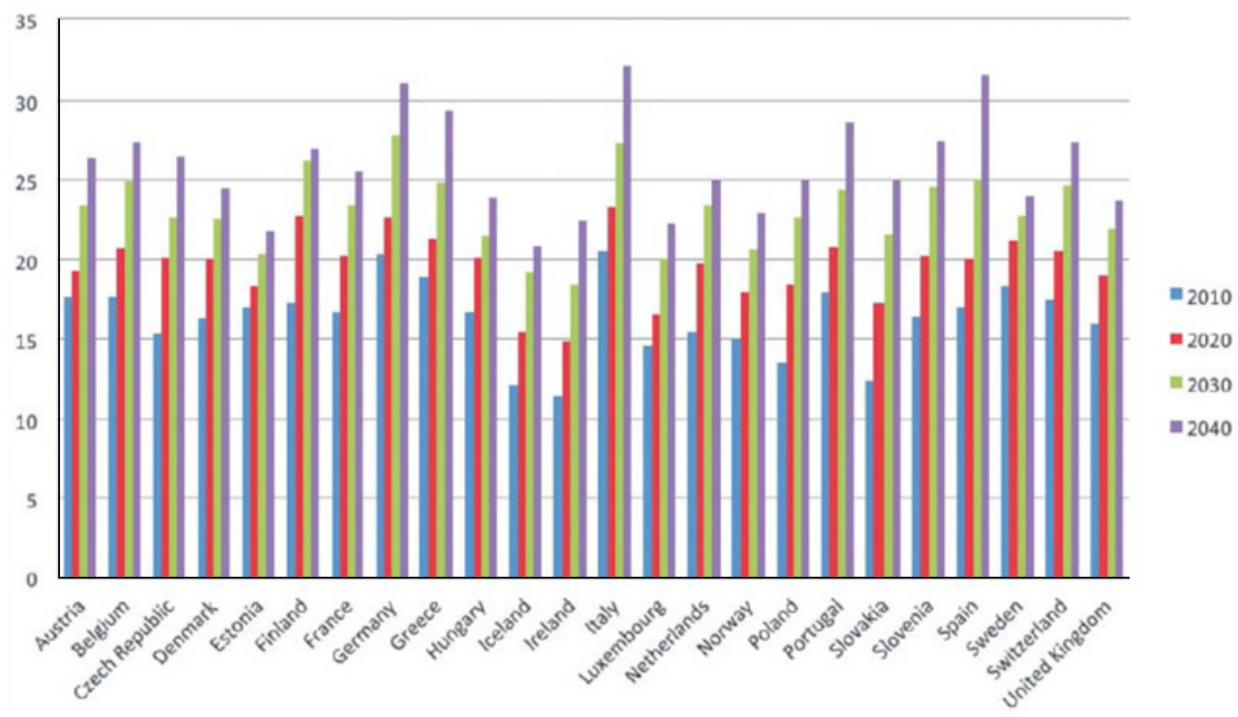

Fig. 3. Projected Percentage of Population aged 65 Years and over (per country).

Figure 3 depicts the geographical pattern in growing elderly population: the first countries to reach it are mostly the Northern European countries, followed by the Western and Southern Europe countries; and eventually, Eastern Europe countries are expected to reach the higher speed of increase only towards mid-century. Additionally, if we do not consider the median age indicator, the countries that are most likely expected to experience the earliest demographic changes include central and eastern European member states such as Germany, Poland, Romania and Slovakia, the first of these about ten or twenty years earlier [9]. At the same time, this is translated into an increase of the number of patients that face chronic health diseases.

\subsection{Description of the Croatian pilot}

The Croatian pilot study took place on the site of Psychiatric hospital "Lopača" and the homes for the elderly located in Rijeka, with the cooperation of the project partners City of Rijeka and Croatian Health Insurance Fund. The main target group in the Rijeka Pilot are people over 65 suffering from mental health diseases or other mental health disorders.

In Rijeka City, according to the last census the population was about 141.000 , with $17 \%$ of citizens older than 65 years. In addition, the number of the people suffering from the mental health diseases and mental health problems increased by more than $50 \%$ in the last 10 years. Before INCA pilot, there was an insufficient coordination within health care (Primary, Secondary, Tertiary) as well as between social care and health care.

Main problems, addressed in the pilot study, included the evidence for existing healthcare and mental health, and health and social service models that aim to encourage integrated working. The study dealt with practical difficulties faced, and how they might be overcome to improve the level of mental care service in the area of Rijeka, and to improve health and quality of life of the people suffering from mental health problems or disorders. The project used an extensive questionnaire (about 130 questions) to identify patients to be involved in the pilot and gather all the required information about them. 


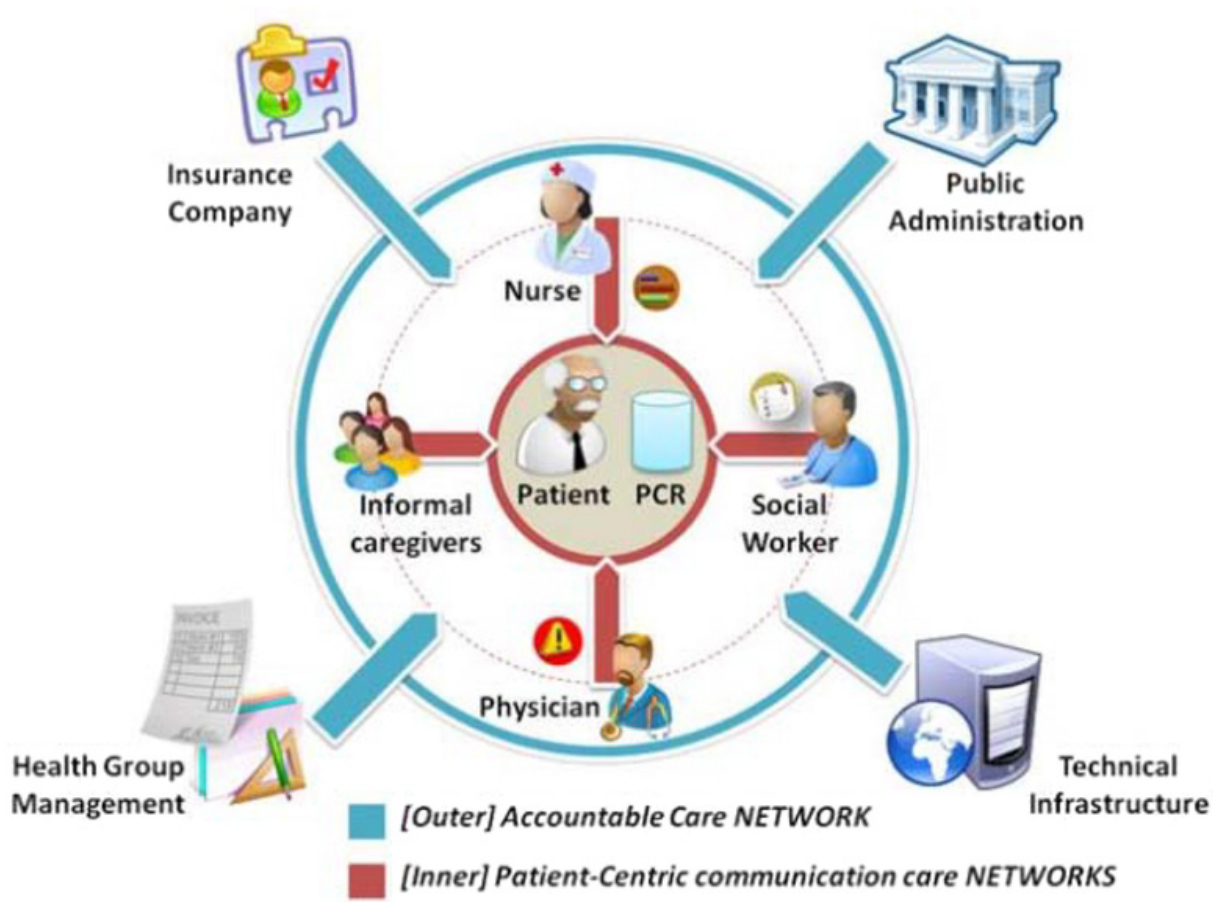

Fig. 4. Four inter-related cornerstones of Project INCA.

\section{Material and methods}

\subsection{INCA's 4 inter-related cornerstones}

Project INCA introduced benefits for end users and realized the concept of patients as coproducers of health as an integral part of their entire integrated care Network and the "GoBetween" hub among all the care providers, however, in a very singular and innovative way, as depicted in Fig. 4 [10].

INCA pilots have introduced a novel concept which combines an [Outer] Accountable Care Network of [Inner] Patient-Centric communication care Networks. The inner "patientcentric Network of persons and devices" is the main building block of the new core of eHealth service provision. Patient is the owner and stays in the centre, granting and removing permits to the rest of members or devices of his/her network and taking on a greater responsibility and more active role in managing their own wellbeing. This is done in a more collaborative partnership with care providers: formal and informal care providers that have access to Patient information and/or interchange information with Patient and other care providers throughout his/her Patient Care Repository (PCR). The outer Network composed by Accountable Care Agreement deploying partners oversees assuring better coordination of all the stakeholders serving inner "patient-centric Networks, while reducing healthcare costs and with any kind of metrics for supporting better decisions. Here, the role of analytics and Business Intelligence is critical to provide insights into the wealth of data collected. Further, this data will be also critical to ensuring the economics of the Accountable Care Agreement is sound and cost savings are distributed equitably amongst the providers concerned. 


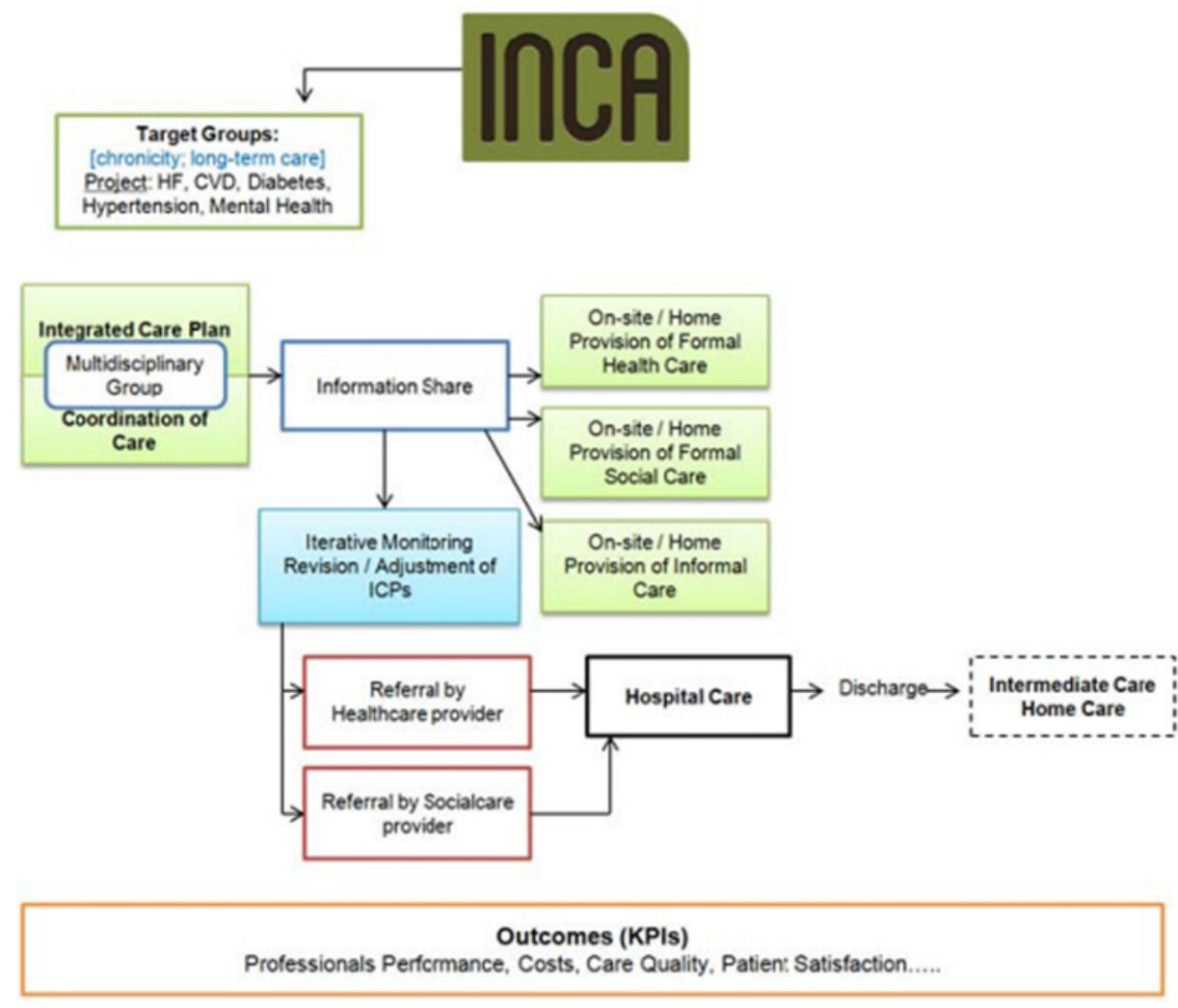

Fig. 5. INCA pathways for integrated care.

\subsection{Care pathways/care actions}

Utilizing the cloud-based ICT tool, we have developed an integrated e-care model and introduced personalised care pathways (as shown on Fig. 5.) for the elderly people suffering from various health problems (including heart failure, cardio-vascular diseases, diabetes and mental health issues). This model employs a patient-centric approach to care delivery, giving the patient (or the person acting on behalf of the patient) power to control (and to share) any data relevant to treat patient's health condition.

Initially, ten KPI's were considered for the use in the pilots:

1. Mortality rate

2. Hospital admissions rate

3. Hospital readmissions rate

4. Hospital stays: number of days of stay and average length of stay

5. Use of drugs by the type of drug

6. Consultation: Number of family medicine consultations (face-to-face, at home) and speciality

7. Emergencies: Number of visits

8. Patients' health auto-assessment: to allow the calculation of quality adjusted life years (QALYs) 
Table 1. KPI's comparison at baseline (Q3-2014) and after the INCA Pilot (Q1-2016).

\begin{tabular}{|l|c|c|}
\hline KPI & Baseline (Q3-2014) & After INCA Pilot (Q1-2016) \\
\hline MHDs Mortality Rate & 1.31 & 1.25 \\
\hline MHDs Admission Rate & 1.84 admissions per patient/year & 1.80 admissions per patient/year \\
\hline $\begin{array}{c}\text { MHDs Re-Admission } \\
\text { Rate after 30 days }\end{array}$ & $1 \%$ of patients are re-admitted & $1 \%$ of patients are re-admitted \\
\hline $\begin{array}{c}\text { MHDs Re-Admission } \\
\text { Rate after 6 months }\end{array}$ & $8 \%$ of patients are re-admitted & $7.6 \%$ of patients are re-admitted \\
\hline $\begin{array}{l}\text { MHDs Re-Admission Rate } \\
\text { after 1 year }\end{array}$ & $13 \%$ of patients are re-admitted & $13.4 \%$ of patients are re-admitted \\
\hline MHDs Stays (average length) & 29 days per patient/year & 26 days per patient/year \\
\hline MHDs Visits to GP (PCP) & 23.66 per patient/year & 31.33 per patient/year \\
\hline MHDs Visits to Psychiatrists & 11.11 per patient/year & 12.13 per patient/year \\
\hline $\begin{array}{l}\text { Health Visitors/Community } \\
\text { Nurses visits to MHDs }\end{array}$ & 13.8 per patient/year & 15.4 per patient/year \\
\hline Provider's Satisfaction & 3.20 (out of 5) - Average & 3.90 (out of 5) - Very good \\
\hline
\end{tabular}

9. Changes in lifestyle: can be controlled through indicators such as control of sedentary lifestyle, obesity control, etc.

10. Levels of satisfaction: both professionals and patients.

\section{Results and discussion}

We have used INCA's Care Manager Interface to create one Care Program: Health and Social Care for the patients/care users with Mental Health diseases or other Mental Health disorders. The main goal was to improve accessibility of Health and Social Services by using INCA for coordination of the providers of Social and Health Services. Patient's classification relies on an active collaboration between sanitary and social providers, as patient's classification results from combining information already in hands of medical and social providers.

In this implementation, there are significant legal problems when it comes to the interchange of social and medical information. The trusted mediator/contact point for the patient that could legally have access to medical as well as social information is the family doctor. However, until now whenever the family doctors were supposed to take over tasks that are related with the patients' social well-being, they were reluctant to implement them, as they thought that it was not part of their job.

On the other side, due to legal reasons, the social side cannot be the trusted single contact point, since social workers are not legally qualified to receive medical information, not even with the patient's consent. To overcome this obstacle, the family doctors are now receiving financial incentives to take over social tasks, paid on a per task basis. It is important to emphasise that on this pilot we have not measured neither Quality of Life (QoL) nor Patient Satisfaction (PS), nor costs. However, pilot results indicate that the implementation of sociosanitary care pathways makes economic and qualitative sense, as shown in Table 1.

It can be seen that the provider satisfaction increased from 3.2 as measured before INCA to 3.9 measured with INCA. Further, the number of hospital stays due to mental health disorders (MHD) decreased by from 29 days per patient per year to 26 days/patient/per year, while patients more frequently visited their GP and were also more frequently visited 
themselves by their community nurse. This resulted in the better given care for the patients, better perception of the caregivers by both the patient and their family. Increased workload by the healthcare professionals (i.e. family doctors) was compensated through social security funding.

Conducted pilot project has fulfilled its main goal, which was to improve accessibility of Health and Social Services by using ICT based solution to coordinate the providers of Social and Health Services. End users were able to have information on all the medical and social services to which an individual is entitled to leading in turn to the production of an Integrated Care Plan.

\section{Conclusion}

Seen as a whole, INCA has perceived as generally effective and beneficial and has served the Croatian healthcare providers, involved in the project, to take qualified decision in a foreseen public procurement process, which is actively promoted by the Croatian Government that sees "Integrated Care" as one of their focal points in for a sustainable society.

For the socio-sanitary care providers the benefit is the ability to influence clinical outcomes by remotely monitor and coordinate care initiatives. Using this approach government has an effective aid to meet quality thresholds, generate clinical outcome metrics and improve satisfaction of the citizens using health and social care services.

The authors would like to thank the entire "INclusive INtroduction of Integrated Care (IN3CA)" project stakeholders funded through ICT PSP programme.

\section{References}

[1] European Commission, eHealth Task Force Report - Redesigning health in Europe for 2020 (Luxembourg: Publications Office of the European Union, 2012). Available: https://ec .europa.eu/eip/ageing/library/redesigning-health-europe2020_en

[2] OECD, Health at a Glance 2013: OECD Indicators (OECD Publishing, Paris, 2013), Available: https://doi.org/10.1787/health_glance-2013-en

[3] J. Kern, et al., Declaration on eHealth, Academy of Medical Sciences of Croatia, Zagreb. Available: http: //www. amzh.hr/news $\% 20$ and $\% 20$ events .html

[4] M. Harris, F. Greaves, S. Patterson, J. Jones Nielsen, Y. Pappas, A. Majeed, J. Car, J. Ambulat. Care Manag. 35(3), 216-25 (2012)

[5] M. Kovač, Computer 47(10), 34-42 (2014)

[6] European Commission, B3 Integrated Care Action Group. A compilation of Good Practices - Integrated care for chronic diseases (Second edition). Available: http://ec .europa.eu/research/innovation-union/pdf/active-healthyageing/gp_b3.pdf

[7] IN3CA project. Available: http://www.in3ca.eu

[8] European Commission, eHealth Action Plan 2012-2020, Available: http://ec. europa.eu/information_society/newsroom/cf/dae/document.cfm?doc_id= 4188

[9] A. Džakula, A. Sagan, N. Pavić, K. Lončarek, K. Sekelj-Kauzlarić, Croatia: Health Sys. Rev. Health Syst. Transit. 16(3), 1-162 (2014)

[10] I. Ljubi, S.B. Romac, H. Belani, Towards Cloud-Based Integrated Socio-Sanitary Care e-Services in Croatia, International Conference on the Learning Health System in Europe (2015) 